\title{
Internacionalización y reforma del sector de seguridad y defensa en Colombia ${ }^{1}$
}

\author{
https://doi.org/10.21830/9789585350601.03
}

\author{
Martha Lucía Bahamón Jara \\ Ximena A. Cujabante Villamiß \\ Mateo Morales Callejas ${ }^{4}$ \\ Universidad Militar Nueva Granada
}

\section{Resumen}

El capítulo aborda los principales retos y tendencias en relación con el proceso de reforma del sector seguridad en Colombia. A través de una revisión de las principales discusiones en la materia, se sugiere que algunos temas que debe enfrentar el país tienen que ver con la ausencia de una normativa clara de seguridad y defensa; la confusión de funciones entre policías y militares; la existencia de un distanciamiento entre la sociedad civil y los sectores de la seguridad y la defensa, y la falta de atención a las necesidades

1 Este capítulo es producto de investigación del proyecto INV-EES 2975 de 2019 "El panorama del post conflicto en Colombia: un acercamiento desde la gobernanza y la reforma del sector seguridad", financiado por la Universidad Militar Nueva Granada. Se reconoce la participación de la Universidad del Rosario en el marco del Convenio Especial de Cooperación con la Universidad Militar Nueva Granada. Las secciones de este capítulo relacionadas con los retos de Colombia frente a la reforma y la gobernanza del sector seguridad, así como con la cooperación internacional en seguridad, se basan en una serie de trabajos desarrollados por Arlene B. Tickner, profesora titular de la Facultad de Estudios Internacionales, Políticos y Urbanos de la Universidad del Rosario, en colaboración con Mateo Morales, coautor de este libro. El uso de dichos trabajos, que están referenciados a lo largo del capítulo, cuenta con la aprobación expresa de ambos autores.

2 Abogada de la Universidad Libre de Colombia, especialista en Derecho Administrativo de la Universidad Santo Tomás y magíster en Defensa de los Derechos Humanos y Derecho Internacional Humanitario. Exvicerrectora académica de la Universidad Militar Nueva Granada en el periodo 20092015. Directora de Posgrados de la Facultad de Relaciones Internacionales, Estrategia y Seguridad. Docente tiempo completo de carrera. Asesora y consultora de instituciones de educación superior. ORCID: https://orcid.org/0000-0002-5877-6886. Contacto: martha.bahamon@unimilitar.edu.co

3 Politóloga de la Universidad Javeriana, especialista en Negociación y Relaciones Internacionales de la Universidad de los Andes, magíster en Asuntos Internacionales y doctora en Estudios Políticos de la Universidad Externado de Colombia. Docente de tiempo completo de la Facultad de Relaciones Internacionales, Estrategia y Seguridad de la Universidad Militar Nueva Granada. Orcin: https://orcid. org/0000-0002-5473-163X. Contacto: ximena.cujabante@unimilitar.edu.co

4 Politólogo y magíster en Ciencia Política de la Universidad de los Andes. Orcid: https://orcid. org/0000-0001-5272-1959. 
territoriales en materia de seguridad. Igualmente, se propone la internacionalización como un elemento adicional que debería ser considerado en la reflexión conceptual sobre las reformas al sector seguridad. El capítulo evidencia cómo la internacionalización es un componente visible en la práctica colombiana de reformar su sector de seguridad y que cuestiona ciertos presupuestos teóricos del concepto.

Palabras clave: Colombia; defensa; internacionalización; posacuerdo; retos; seguridad.

\section{Introducción}

Con ocasión de la firma del acuerdo de paz entre el gobierno de Colombia y las FARC se ha comenzado a desarrollar una importante discusión frente a la necesidad de generar una reforma al sector seguridad del país. Además del cambio de las funciones de las Fuerzas Armadas y su acomodo a las nuevas dinámicas planteadas por el posconflicto, la necesidad de reorientar el gasto público hacia el desarrollo y de someter las actividades de seguridad y defensa al debate y el control civil ponen de presente la importancia de reflexionar sobre el futuro de este sector en Colombia.

Pese a la relevancia del caso, muy poco es lo que se ha explorado frente a las necesidades de reforma y gobernanza de la seguridad, empezando por saber si existe una hoja de ruta que indique el camino que se debe seguir. La literatura sobre gobernanza y reforma del sector seguridad (GRss) ha sido clara en señalar que estos procesos no surgen de la noche a la mañana ni de la improvisación. Para generar una reforma exitosa del sector seguridad y defensa en el país se debe comenzar por poner en el debate público este tema de vital relevancia, hacer un diagnóstico del sector y estipular democráticamente los horizontes hacia los cuales se debe transitar.

En ese orden de ideas, el presente capítulo pretende generar algunos insumos iniciales frente a esta materia. De un lado, se expondrán brevemente algunos de los principales retos que enfrenta este proceso en Colombia, con especial énfasis en (1) la ausencia en Colombia del debate público en torno a los temas de seguridad y defensa; (2) la falta de liderazgo civil y de una política pública de seguridad y defensa que oriente el rumbo del sector; (3) las relaciones cívico-militares y su adaptación a las particularidades nacionales; 
(4) la falta de claridad en las funciones de los distintos servicios de las Fuerzas Armadas (en especial, militares y policías) a raíz del desarrollo del conflicto armado en el país, y (5) el lugar central que tiene el factor territorial a la hora de hablar de la seguridad en Colombia.

De otro lado, el capítulo explora cómo la internacionalización también se puede entender como un elemento clave dentro de la Grss. Al respecto, se sugerirá que la internacionalización, entendida como la estrategia consciente y direccionada en función del cumplimiento de objetivos concretos, puede ser una cara relevante de los procesos de Grss que realizan los países en transición, tales como Colombia. Sin embargo, existen otras formas de internacionalización, no planeadas, por medio de las cuales ciertos efectos de lo internacional se hacen sentir y generan importantes repercusiones en relación con el sector de seguridad y defensa. Se pretende que esta última idea constituya un complemento frente a los argumentos que sugieren que todo proceso de reforma es estructurado y planeado.

Por medio de ejemplos tales como la adopción de normativas internacionales de Derechos Humanos y del enfoque de género, el capítulo argumenta que no se puede considerar necesariamente como positivo todo proceso de reforma del sector que surge como resultado de la transición de la guerra a la paz, en el sentido de efectivamente lograr mayor gobernabilidad y cercanía entre el sector civil y el militar, ni que ha sido impulsado por las autoridades civiles.

\section{Reforma del sector seguridad en Colombia}

La Grss se entiende como la creación de esquemas de buen gobierno en el sector seguridad que incluyen la promoción de la efectividad y la eficiencia en el suministro de la seguridad y la justicia, la transformación de las funciones de las Fuerzas Armadas acorde con las necesidades de la ciudadanía, la construcción de una relación cívico-militar fluida, el fortalecimiento del control civil sobre el sector y el mejoramiento de las capacidades de los civiles de incidir en la toma de decisiones en seguridad y defensa, todo dentro del marco de la democracia, el Estado de derecho, la transparencia, la rendición de cuentas y el respeto por los Derechos Humanos. Por lo tanto, se comprende que la Grss 
constituya un tema de fundamental relevancia de cara al proceso de transición que atraviesa el Colombia.

Pese a su importancia, se aprecia que no existe un debate público sobre hacia dónde debe transitar un sector que ha sido clave en la historia reciente de Colombia. Esta situación no deja de ser paradójica, si se tiene en cuenta que el país ha atravesado más de medio siglo de conflicto, tiempo durante el cual los asuntos de seguridad y defensa han sido determinantes. Pese a ello, las discusiones y los asuntos de seguridad y defensa han quedado relegados en las manos de unos pocos expertos que se encargan de su manejo, conceptualización y ejecución.

Por supuesto, esta falta de debate público ha redundado o ha sido el resultado de otra serie de retos que Colombia debe enfrentar para desarrollar un efectivo proceso de reforma de su sector seguridad y defensa. Por una parte, es necesario mencionar la ausencia de un claro liderazgo civil y de una política nacional de seguridad y defensa. A la falta histórica de presencia estatal a lo largo y ancho de la geografía nacional, por otra parte, se le suma otra falencia de larga data: la apropiación incompleta por parte del Estado colombiano y de la clase gobernante de los temas de seguridad y defensa (Morales \& Tickner, 2019).

Esta separación entre lo político y lo militar se remonta al gobierno de Alberto Lleras Camargo (1958-1962), en el cual se generó una distinción tajante entre un ámbito y el otro con el fin de evitar el involucramiento excesivo de los civiles en la conducción de lo militar, y de los militares en la conducción de la política. La así llamada “doctrina Lleras” permeó el discurso de las relaciones cívico-militares durante más de tres décadas (Leal, 2002, p. 37) y se convirtió en un dogma, el cual se vio reflejado en la falta de una política de Estado respecto de la conducción del conflicto armado y que aún en la actualidad se observa en la falta de una fuerte guía civil respecto a la reforma del sector seguridad con miras a la construcción de la paz.

Esto ha generado dificultades considerables en el manejo del conflicto armado, el orden público y ahora la reforma del sector seguridad. Así mismo, ha repercutido en qué tanto la institución militar y la policial han desarrollado sus propios planes operacionales con manifestaciones muy variadas, dada la 
vasta geografía del país y la forma diferencial como el conflicto armado y los problemas de seguridad se han manifestado en el territorio nacional. Debido a la presencia variable del Estado y la ausencia de liderazgo civil, las fuerzas de seguridad han terminado por sustituirlo en muchas de sus funciones, lo que ha producido distorsiones importantes en cuanto al papel que deben desempeñar.

El hecho de que las distintas ramas de la fuerza pública estén llevando a cabo sus propios procesos de repensarse en función del posconflicto trae otro tipo de problemas relacionados con la ya mencionada falta de presencia estatal. A saber, el hecho de que se trate de apuestas de las mismas instituciones de seguridad y defensa para generar acciones integrales más allá de sus funciones tradicionales puede fomentar negativamente que el Estado haga presencia en toda la geografía del país. Por consiguiente, esto no solo produce una sobrecarga en funcionarios e instituciones que constitucional y misionalmente no deberían dedicarse a ciertas acciones, sino que además fomenta el desentendimiento por parte de la dirigencia civil.

En ese orden de ideas, lo que se aprecia en Colombia es la carencia de un norte sobre cómo superar esta ya arraigada problemática y consolidar una política pública de seguridad, actualizada y contextualizada a las realidades del país. Ejemplo de ello es la inexistencia de una ley de seguridad y defensa, así como de una estrategia nacional de seguridad. En contraste, un proceso de reforma y gobernanza de la seguridad solo se puede dar si hay apropiación nacional y liderazgo civil que construya sobre las iniciativas que ya están gestando las fuerzas, pero que también cuente con un claro direccionamiento estatal (Morales \& Tickner, 2019).

Otro importante reto que debe enfrentar el país de cara a la Grss tiene que ver con lo que se puede denominar como la "variable territorial". Esto quiere decir que a la hora de diseñar políticas públicas de seguridad es crucial tener en cuenta la gran diversidad regional que caracteriza al país, pues no es posible ni deseable diseñar una política de talla única para todo el territorio nacional. Es muy problemático mantener las discusiones sobre la seguridad solo en el nivel nacional y en las principales ciudades del país, teniendo en cuenta que las dinámicas del conflicto y el posacuerdo tienen mayor impacto en lo local. 
Las variables locales, además de afectar la formulación de una ley de seguridad y defensa, también impactan el grado de complementariedad entre los distintos servicios de las Fuerzas Armadas. Las amenazas a la seguridad no se presentan de manera homogénea en toda la geografía, sino que varían regionalmente y por lo tanto involucran a actores violentos no estatales, lógicas de interacción y problemáticas diferentes. Si bien ejercer control social, político y de seguridad es lo que se requiere en términos genéricos, la aplicación de políticas de forma indiferenciada puede generar zonas grises y solapamiento entre funciones, por lo cual es importante sopesar cuál es el valor agregado de cada fuerza y por qué puede contribuir más que otras en territorios particulares.

En tercera medida, la enorme confusión de funciones que existen en la fuerza pública es otro importante reto que debe afrontar Colombia, ya que este ha sido un asunto particularmente complicado a raíz de que la Policía se ha involucrado paulatinamente en el conflicto armado y, subsecuentemente, se ha militarizado, mientras que se ha producido la "policialización" del Ejército (Morales \& Tickner, 2019). Esta confusión de funciones ha generado tensiones que transitan desde lo nacional a lo local, impactan en el presupuesto y afectan la administración efectiva de la seguridad. Con esto se quiere decir que más allá de discutir si se debe privilegiar una doctrina de seguridad nacional (militar) o una de seguridad ciudadana (policial), la cuestión está en cómo ambas lógicas deben ser entendidas como parte de la reforma del sector seguridad, pues las dos impactan de manera diferenciada en distintas partes del territorio nacional.

Si bien la separación de funciones es una condición necesaria de la reforma del sector seguridad, también es crucial que simultáneamente exista complementariedad entre las fuerzas, dada la complejidad de los problemas de seguridad que se presentan en el mundo contemporáneo y en un país como Colombia, que si bien está solucionando el enfrentamiento armado con las FARC, está lejos de ser un escenario de paz dada la presencia de múltiples otros actores armados.

Muy vinculado con los anteriores factores, un cuarto componente clave que se debe abordar con respecto a la Grss en Colombia son las llamadas relaciones cívico-militares, que en esencia hacen referencia, por un lado, al tipo de relaciones que establecen los sectores civiles gubernamentales y el esta- 
mento militar, y, por otro, a la forma como sus interacciones tienden a variar a partir del mayor o menor grado de autonomía y subordinación de los militares con respecto al sector civil (Andrade, 2012). De manera general, los diferentes estudios sobre el caso colombiano argumentan que las relaciones entre civiles y militares se han caracterizado por una separación entre las dos esferas a raíz de la doctrina Lleras, lo cual ha producido una oscilación constante entre acercamiento y alejamiento de ambas partes, dependiendo de las políticas del gobernante de turno. En últimas, esto ha conducido a un manejo errático de la seguridad y la defensa del país debido a la carencia de una política pública de Estado, como se sugirió anteriormente (Andrade, 2012; Leal, 2002).

Sin embargo, el asunto de las relaciones cívico-militares en Colombia supera o amplía el enfoque dedicado exclusivamente a analizar las relaciones entre la élite civil gobernante y las fuerzas de seguridad del Estado. En efecto, cuando se habla de lo civil, no se debe reducir únicamente al sector gubernamental, sino que debe abarcar e incluir a las comunidades, a la sociedad civil ampliada, pues esta ha sido la víctima principal de los estragos del conflicto.

De igual manera, cuando se hace referencia a la transición de la guerra a la paz y a una reforma del sector seguridad, es fundamental normalizar las relaciones con estos actores y no solo con el sector político (civil) (Morales \& Tickner, 2019). En términos comparativos, las relaciones entre la clase política colombiana y los militares no han sufrido los mismos traumatismos de otros países, como Chile o Argentina, donde el estamento militar entró a controlar directamente el Estado, ya que aquí —aunque en algunas ocasiones más y en otras menos - siempre han estado subordinados al poder civil. Por lo anterior, la reflexión se debe desplazar hacia la revisión de las relaciones con la sociedad civil, en especial aquella donde el conflicto armado se ha manifestado con mayor intensidad.

\section{La internacionalización de la seguridad y la defensa}

Además de los argumentos esbozados más arriba referentes a los retos que se deben tener presentes de cara a una reforma de seguridad en el país, vale la pena también poner en consideración un factor que usualmente se tiende a dejar de lado o se da por sentado en las explicaciones relacionadas con la Grss. 
Concretamente, se hace referencia al factor de la internacionalización como un posible dinamizador o instrumento de dicho proceso.

La internacionalización se puede entender de dos maneras distintas: por una parte, como el impacto no planeado de diversos factores internacionales sobre la conducta y la normativa que caracteriza al sector seguridad y defensa. Por otra, como una estrategia intencional y planeada por parte del sector seguridad y defensa para proyectar su quehacer hacia distintos espacios mundiales. Tal vez la segunda acepción es la más clara cuando se hace referencia al término y se vincula también de forma más directa con los procesos de reforma del sector seguridad. Es común que en las sociedades en transición de la guerra hacia la paz deba existir una reorganización y disminución del pie de fuerza que fue empleado durante el conflicto, como una manera no solo de reducir el gasto, sino también como una muestra clara de que las hostilidades han finalizado. Una manera de reorganizar a las fuerzas consiste, precisamente, en que se utilicen en esquemas de cooperación internacional, de manera bilateral o multilateral.

Este ha sido el caso de Colombia, que desde 2010 ha potenciado e institucionalizado una política de cooperación internacional en materia de seguridad con miras a posicionar al país como exportador de experticia en diversos temas relacionados con el conflicto armado y el crimen organizado. Lo que el Ministerio de Defensa llama la diplomacia de la seguridad se ha concentrado hasta ahora en América Latina y el Caribe, pero también se ha proyectado hacia África Occidental, así como hacia organismos multilaterales como la Organización de Naciones Unidas (ONU), la Unión Europea y la Organización del Tratado del Atlántico Norte (OTAN). Por lo tanto, no solo consiste en una estrategia de visibilización y reputación internacional, sino también de reorientar el recurso humano altamente profesionalizado en actividades similares en el exterior.

En contraste, cuando se aborda la internacionalización en su primera interpretación, es decir, como efectos no planeados de lo internacional, pareciera que esta no fuera tan evidente ni se relacionara de manera tan estrecha con los procesos de reforma al sector seguridad. Sin embargo, el caso colombiano pone de presente que, así entendida, la internacionalización también ha 
cumplido un papel clave en las fuerzas de seguridad del país. Específicamente, ha contribuido a que la fuerza pública introduzca e interiorice las normativas de Derechos Humanos y Derecho Internacional Humanitario, así como que el sector de seguridad y defensa del país haya incorporado las consideraciones de género.

Con respecto a lo primero, hoy en día las fuerzas de seguridad en Colombia son absolutamente conscientes de que deben circunscribir sus actuaciones a los estándares de estas normativas, una situación muy distinta a la que se presentaba hace diez años. El tema de género, por su parte, fue introducido mucho más tarde en el país, principalmente a raíz del acuerdo de paz, y aunque sus avances han sido palpables en las fuerzas y el sector de seguridad y defensa, aún se requieren esfuerzos, compromisos y voluntades. De esta manera, tanto las normativas de Derechos Humanos como la inclusión de un enfoque de género en las fuerzas de seguridad han sido entendidas como componentes esenciales de los procesos de reforma. Sin embargo, valga aclarar que en Colombia la llegada e inclusión de la normativa de los Derechos Humanos no se dio como resultado de un proceso de construcción de paz, sino que sucedió de forma previa durante y como resultado del desarrollo del conflicto armado. Además, si bien la adopción de consideraciones de género es parte de las recomendaciones en materia de Grss, en Colombia su desarrollo ha sido escaso y ha correspondido más a los esfuerzos propios de las fuerzas de seguridad que a una estrategia planeada y estructurada desde la dirigencia civil.

En lo que resta del capítulo se desarrollan estos casos de internacionalización, en cuanto se pueden considerar como formas o herramientas de los procesos de reforma al sector seguridad.

\section{Internacionalización por cooperación}

Si bien la idea de Colombia como exportador de seguridad puede parecer una tendencia novedosa vista desde dentro e incluso a nivel regional, no lo es en términos de las dinámicas que se están generando en el mundo en relación con el sector seguridad y defensa. Al decir de autores como Schnabel y Ehrhart (2005, p. 3), no solo los ejércitos de los países en posconflicto sufren 
actualmente procesos de transformación, sino que esto opera incluso en las democracias más avanzadas del planeta, donde los denominados militares posmodernos ya no se limitan exclusivamente al mantenimiento de la defensa nacional, sino que se han visto involucrados en una enorme cantidad de actividades que previamente escapaban a sus funciones normales. En ese sentido, el soldado moderno, y por extensión la Policía, ya no son solo combatientes, sino que son constructores de paz, trabajadores sociales, diplomáticos y trabajadores de cuerpos de paz. Pero, además de esto, la internacionalización de las fuerzas también se ha vuelto una tendencia de los ejércitos posmodernos. Esto ocurre de manera bilateral, proveyendo de asistencia técnica a contrapartes, pero sobre todo en términos multilaterales por medio de espacios como la OtAn o Naciones Unidas.

Desde comienzos del siglo XXI, Colombia ha recibido un número creciente de solicitudes y ha aumentado su oferta de cooperación en seguridad a distintos gobiernos en toda América Latina y el resto del globo. Hasta la primera elección de Santos en 2010, las solicitudes que llegaban a Colombia para la cooperación en seguridad fueron tratadas de manera desestructurada (Tickner, 2014).

Con la llegada de Santos, estas acciones adquirieron un talante más estratégico y se convirtieron en parte de la política exterior del país. A su vez, se incorporó el tema al Plan Nacional de Desarrollo 2010-2014, "Prosperidad para todos" mediante la creación de la Estrategia Nacional de Cooperación Internacional 2012-2014. En este sentido, se concretó la Estrategia de Cooperación Internacional en Seguridad Integral, coordinada por la Cancillería, encargada de interactuar con los gobiernos extranjeros y coordinar los esfuerzos específicos de cooperación con el Ministerio de Defensa Nacional, la Policía (PNC) y la Agencia Presidencial para la Cooperación (APC). Colombia ofrece un portafolio de servicios "a la carta" en siete áreas: (1) desarrollo organizacional, (2) lucha contra el narcotráfico, (3) crimen organizado, (4) seguridad ciudadana, (5) lucha contra la corrupción, (6) Derechos Humanos y Derecho Internacional Humanitario y (7) capacidades operativas. Además, adapta la cooperación a las necesidades de cada solicitante a través de un diagnóstico inicial sobre la seguridad y las capacidades institucionales del receptor, la plani- 
ficación y recaudación de fondos, la ejecución, y el seguimiento y la evaluación (Tickner \& Morales, 2105, p. 194).

El crecimiento exponencial en la cooperación triangular entre Colombia y Estados Unidos confirma su importancia como mecanismo conjunto que satisface los intereses respectivos de política exterior de los dos países. Entre 2013 y 2014 el número de actividades contempladas en el Plan de Acción aumentó de 36 a 145, y en 2015 creció hasta 319. Se entrenó un total de 7.743 miembros de las instituciones de seguridad y defensa de los países centroamericanos y del Caribe en ese periodo (Tickner, 2016). Desde la perspectiva de Washington, y en seguimiento de la estrategia de "entrenar a entrenadores", en la medida en que Estados Unidos "lidera desde atrás", apoyando a Colombia como hacedor de seguridad, Colombia le ayuda a capacitar a otros, con lo cual genera un efecto multiplicador en aquellos países de Mesoamérica y el Caribe considerados problemáticos en términos de la inseguridad y la violencia. Por su parte, el interés colombiano apunta simultáneamente a madurar la asociación estratégica con el país del norte y aumentar los niveles propios de influencia en el hemisferio. Así, "seguir desde adelante" a Estados Unidos le permite acrecentar los grados de reciprocidad en la relación bilateral y desarrollar una agenda más ambiciosa de política exterior (Tickner \& Morales, 2015, p. 198).

En el centro de la cooperación triangulada colombo-estadounidense está la idea de que Colombia tiene instalaciones, equipos, know-how y entrenadores que pueden ser utilizados a un menor costo económico, con resultados potencialmente mejores y sin los riesgos políticos asociados con una presencia militar directa de Estados Unidos (Tickner, 2016). Un tercer objetivo que explica la intensificación de la cooperación Sur-Sur y triangular en seguridad en años recientes está relacionado con la búsqueda de actividades alternativas para las Fuerzas Armadas en un escenario de posconflicto. Un análisis reciente de la Washington Office on Latin America (WoLA) sugiere que la reforma del sector de la seguridad después de la firma de los acuerdos de paz con la guerrilla conllevará, entre otros, una reducción sustancial del tamaño actual de las Fuerzas Armadas (Isacson, 2016).

Esta diplomacia para la seguridad no ha desaparecido durante la administración de Iván Duque, pese a las claras diferencias existentes entre ambos 
gobiernos frente a asuntos neurálgicos del país. De hecho, con algunas variaciones los objetivos coinciden grosso modo con los lineamientos generales de la política exterior de Santos, tales como posicionar al país como líder y referente hemisférico y global de la cooperación en la lucha contra el terrorismo, el crimen organizado transnacional y el problema del narcotráfico; aumentar su incidencia multilateral en dichas temáticas; fomentar la industria nacional de defensa, y promover la legitimidad de los militares y la Policía mediante el respeto a los Derechos Humanos y la aplicación del Derecho Internacional Humanitario.

Según queda claro a partir de la revisión de la Política de Seguridad y Defensa de la administración Duque, la estrategia de cooperación en seguridad no solo pretende aumentar la capacidad disuasiva de la fuerza pública hacia dentro y hacia fuera, sino que también tiene el propósito de constituirse como una herramienta que eleve el perfil de Colombia en el plano internacional y la ponga a dialogar como iguales con "potencias aliadas", con las cuales se comparten intereses de seguridad comunes. Esto último, por supuesto, haciendo referencia a la relación estratégica que desde la era Santos se ha estado consolidando con Estados Unidos, en donde Colombia, lejos de ser un actor secundario, ha venido liderando la cooperación en materia de seguridad.

Igualmente, es de destacar la voluntad del país de seguir participando en misiones de paz, situación que ya había quedado planteada durante la administración Santos de enviar cinco mil tropas para poner al servicio de Naciones Unidas. Para algunos analistas, la participación de las fuerzas colombianas en Operaciones Multilaterales de Mantenimiento de la Paz (OMP) puede constituirse en una estrategia para conservar el personal y asegurar el mantenimiento de los equipos adquiridos en un escenario de posconflicto.

No cabe duda de que las fuerzas de seguridad de Colombia han atravesado por un importante proceso de modernización, profesionalización y adquisición de conocimiento, que ha sido documentado por una gran diversidad de estudios. De una parte, militares y policías se han vuelto expertos en el combate de guerra irregulares, con presencia de múltiples actores, economías ilícitas y crimen organizado. De otra parte, como se verá más adelante, han transitado por un importante proceso de concientización e interiorización del 
respeto por los Derecho Humanos, el respeto por las víctimas y la generación de garantías en la legitimidad e institucionalidad para llevar a cabo procesos de desmovilización y reinserción de grupos armados irregulares (Álvarez \& Duque, 2020, p. 100).

No obstante, vale la pena tener presente que aunque nuestras fuerzas de seguridad saben operar en contextos VICA (Volátiles, Inciertos, Complejos y Ambiguos), como ellos mismos los denominan, eso no quiere decir que en la actualidad estén siendo exitosos frente a la presencia y proliferación de actores armados a lo largo del territorio nacional, donde antiguamente operaban las FARC. Asimismo, la participación en operaciones internacionales no deja de plantear retos frente a los cuales nuestras fuerzas no están capacitadas, teniendo en cuenta que los escenarios donde actualmente se despliegan las OMP se caracterizan por conflictos religiosos y étnicos, que requieren otro tipo de sensibilidades. Según lo reportan Álvarez y Duque (2020), entre 2018 y 2019 la Fuerza de Tarea Colombia se ha estado capacitando precisamente en torno a estos nuevos retos, sumado a temas de Derechos Humanos y al funcionamiento como tal de las operaciones lideradas por la ONU. Habrá que ver en qué medida esta participación, en este caso del Ejército Nacional, será evaluada y monitoreada para extraer lecciones a futuro.

\section{Internacionalización no intencionada: Derechos Humanos y Derecho Internacional de los Derechos Humanos}

A diferencia de los manuales y las guías de operadores, que tratan el vínculo de procesos de Grss con el contexto regional y global de manera somera, los textos y compilaciones académicas ahondan sobre la forma como iniciativas de "control de armas estructural" y salvaguardas socio-políticas que emanan de estructuras globales o regionales empoderan y extienden la participación local de actores de la sociedad civil en materia de seguridad, al mitigar los incentivos o alicientes para el uso de violencia organizada (Cooper \& Pugh, 2002, pp. 23-24). En el caso de la Grss, la literatura académica explica que si bien existen normas relevantes de 
manera tangencial (como es el caso de convenciones, códigos e instituciones internacionales asociadas a la defensa de los Derechos Humanos) o de manera directa (e. g., el Documento Viena de la Organización para la Seguridad y la Cooperación en Europa [Osce]), en cualquiera de los casos la GRss tiene una orientación clara hacia las normas y reglas formuladas a nivel regional y global porque guían el comportamiento de los actores involucrados, sean estas formales o "suaves"/“culturales" (Brzoska, 2011, p. 41).

Más allá de las tendencias regionales, también se puede y debe hacer referencia a las tendencias internacionales y globales relacionadas con que estamos en un mundo cada vez más interconectado y en el que la adopción de normativas surgidas en el seno de organizaciones como Naciones Unidas se vuelve una constante, especialmente en países que se alinean con las visiones de mundo de paz liberal emanadas desde este tipo de organizaciones. Es por esto que temas como el Derecho Internacional Humanitario (Dir) y el Derecho Internacional de los Derechos Humanos (DidH) se convierte en un must para cualquier proceso de reforma del sector seguridad y defensa.

En Colombia el caso tiende a ser bastante particular, ya que al término del conflicto y frente a un complejo escenario de mutación de las amenazas, las fuerzas de seguridad y defensa del Estado han llevado a cabo un extenso proceso de interiorización de dicha normativa (Kalmanovitz, 2018). A diferencia de otros casos en que fuerzas públicas no profesionalizadas inician un proceso de adopción e interiorización de la normativa durante la etapa de posconflicto, en Colombia este proceso se comenzó con intensidad desde la década de los noventa. Sin embargo, esto no emanó de una necesidad de transformación interna del sector seguridad, sino por un tema netamente estratégico vinculado con la ayuda que tanto las Fuerzas Armadas como la Policía recibían de Estados Unidas en función del combate contra las guerrillas y el narcotráfico.

De hecho, para algunos autores (Grabendorf, 2009), en Colombia se viene gestando un proceso de reforma al sector seguridad desde 1989, ligado (y limitado) sobre todo a la profesionalización de las fuerzas de seguridad y a mejorar su imagen de cara a la opinión pública nacional e inter- 
nacional, poniendo a las fuerzas a tono con el respeto de las normativas de Derechos Humanos.

La promulgación de la Constitución de 1991 generó una profunda transformación de esta situación con la idea y el imperativo internacional de "humanizar la guerra”. En ese sentido, se declaró la prevalencia interna de los tratados del Didh y la aplicabilidad del Dir, incluso durante las declaraciones de los estados de excepción. Es importante señalar que todo este contexto de adopción de la normativa estuvo fuertemente marcado por la presencia y presión de actores internacionales, especialmente de los Estados Unidos. A lo largo de las distintas administraciones en Washington desde la década de los setenta, el foco de la ayuda a la fuerza pública colombiana sufrió cambios dependiendo del mayor o menor apoyo que el gobierno de turno diera para enfrentar a los actores insurgentes, dado que la prioridad de los Estados Unidos era combatir las drogas.

La negación por parte de los Estados Unidos de la existencia de vínculos entre la guerrilla y los narcotraficantes, y por ende del término narco-guerrilla —originado paradójicamente en los círculos oficiales estadounidenses 5 -, permaneció constante durante la década de los noventa, con lo cual la ayuda que podía recibir el Ejército se vio seriamente limitada y se priorizó la asistencia a la Policía Nacional (Isacson, 2005; Ramsey, 2008; Tickner, 2000). A esta situación se le sumó el constreñimiento de que la ayuda militar llegara únicamente a las unidades que demostraran un claro cumplimiento frente a las normativas de Derechos Humanos (dada la entrada en vigor de la conocida enmienda Leahy en 1996), en un momento en el que había sospechas de la vinculación de algunos miembros del Ejército con grupos paramilitares y existían denuncias de violaciones a los Derechos Humanos por parte de la institución.

Esto generó grandes frustraciones dentro del Ejército, pues sus integrantes se sintieron abandonados por los dirigentes políticos enfocados en la lucha contra las drogas, mientras que el estamento militar debía controlar la

5 El embajador de Estados Unidos en Colombia durante los ochenta, Lewis Tamps, formuló por primera vez el término narcoguerrilla, que posteriormente se fue generalizando en el discurso militar y político de ambos países (Tickner, 2000). 
otra cara de la situación de orden público que vivía el país, con unas guerrillas que adquirían mayor capacidad bélica debido a su involucramiento en el negocio de la droga. De manera reiterada altos mandos trataron de mostrarle a su contraparte estadounidense que la situación con las guerrillas no admitía una separación entre guerra antiguerrilla y guerra contra las drogas, ya que ambas se estaban fusionando en las FARC (Marcella \& Schulz, 1999; Ramsey, 2008, p. 20). Sin embargo, a raíz de las experiencias de los Estados Unidos en Vietnam y el Salvador al finalizar la Guerra Fría - y por ende la terminación del conflicto ideológico—-, a la necesidad de respetar los estándares de Derechos Humanos y al peligro con el que se leía el problema de los carteles, tanto oficiales del Ejército estadounidense, como miembros del Congreso se negaron una y otra vez a permitir que la ayuda fuera destinada a la guerra contra la insurgencia (Crandall, 2002; Isacson, 2005; Tokatlian, 1988).

Esta situación, relativa a los temas de asistencia y de interiorización de las normativas de Derechos Humanos, comenzaría a modificarse con la entrada en vigor del Plan Colombia. Aunque es cierto que el Plan para la Paz contemplaba una gama amplia de asuntos considerados cruciales para el esfuerzo de paz del gobierno, como la recuperación económica, la reforma a la justicia y la democratización, lo cierto es que el núcleo de la estrategia colombiana era la lucha contra el tráfico de drogas y el fortalecimiento del aparato coercitivo (Tickner, 2007).

Un importante ejemplo de este proceso de interiorización se dio con la promulgación de la Política Integral de Derechos Humanos y Derecho Internacional Humanitario, cuyo propósito consistió en integrar el DiH y el DiDH dentro de las operaciones militares y los mecanismos de control en los ámbitos de las regulaciones, las reformas en los procedimientos militares y frente a los entrenamientos y capacitaciones. De manera fundamental, esta política tuvo el logro de constituir una nueva rama del derecho que se denominó Derecho Operacional, que básicamente constituye una forma de aplicar la normativa internacional al contexto interno de operaciones militares.

Como resultado de la política se dio vida a dos manuales de operaciones (en 2009 y 2015) que operativizan y estructuran los escenarios de aplicación de cada una de las normativas. Por una parte, el DiH se convierte en un marco de regulación para las hostilidades y, por lo tanto, opera como un derecho de 
excepción, mientras que, por otra, el DiDH regula el ámbito de la aplicación de la ley.

Es indudable el avance que ha habido en materia de aceptación e interiorización de las normativas internacionales en materia de Derechos Humanos por parte de las fuerzas de seguridad y defensa del país. Hoy en día estos componentes son parte esencial de la forma de planificar y operar en el territorio por parte de las fuerzas. El conflicto armado y su desarrollo, aunado a las presiones internacionales en términos de respeto de las normativas de DiH y DidH en función de obtener los beneficios de la cooperación en seguridad, fueron promotores tempranos de un proceso de reforma y profesionalización del sector de seguridad y defensa.

\section{Internacionalización no intencionada: género}

Hoy en día sigue siendo molesto, por no decir controversial, hablar de asuntos relacionados con la adopción de un enfoque de género y de garantía de los derechos de las mujeres y las fuerzas de seguridad de los Estados. De hecho, hasta no hace mucho ambos asuntos se veían como un juego de suma cero, en el sentido de que si se implementaba una perspectiva de género y se incluía a las mujeres en los teatros de operaciones, esto implicaba disminuir o que se viera afectada la eficacia y la capacidad de fuego (en el caso de los militares). Por su parte, cualquier esfuerzo por aumentar la eficacia en el plano militar o policial era entendido como un retroceso en la adopción de un enfoque sensible al género, por cuanto se privilegiaba una lógica donde la guerra y la violencia eran los componentes esenciales.

Desarrollos y cambios sociales hacia una mayor autonomía y libertad del individuo, así como hacia la igualdad e integración racial y de género, aunados a maneras diferentes de comprender el tema de la seguridad de forma mucho más amplia y diversa que la supervivencia del Estado (seguridad nacional), han implicado reevaluaciones sobre la relevancia de incluir no solo a las mujeres dentro del pie de fuerza de las instituciones de seguridad y defensa, sino también de adoptar una perspectiva de género tanto en el interior de la organización como en lo externo, es decir, en la forma de implementar el servicio 
militar y policial (Huber \& Karim, 2017). De manera particular, la presencia de personal femenino y la adopción de enfoques diferenciales dentro de las fuerzas se convierten en elementos claves de cara a los procesos de construcción de paz y estabilización de sociedades en conflicto, pues permiten abordar, de una manera sustancialmente distinta, las necesidades y vicisitudes de las poblaciones afectadas por la guerra, así como también de los excombatientes (Bjarnegard \& Melander, 2011; Hudson et al., 2013).

No es extrańo, en este orden de ideas, que la igualdad de género se comprenda como un componente esencial de la Grss. Como se evidenció en el capítulo introductorio, dado que la reforma del sector seguridad implica ante todo reconocer que existen retos de seguridad que exceden por mucho a la seguridad estatal y se vinculan con la seguridad humana, la adopción de enfoques diferenciales, entre ellos el de género, es fundamental para operar de manera eficiente una reforma del sector para lograr una efectiva protección de las (in)seguridades de la sociedad (Centro de Ginebra para la Gobernanza del Sector de Seguridad [DcAF], 2015; Osce, 2016).

Una forma de vincular la reforma del sector seguridad con el género es por medio de lo que se conoce como gender balancing, que consiste en incrementar la cantidad de mujeres en relación con los hombres en todas las organizaciones de seguridad de un Estado. Para ello, se dispone de una serie de acciones tales como la adopción de cuotas obligatorias de personal femenino; la generación de campañas de reclutamiento dirigidas hacia las mujeres; la eliminación de restricciones para que las mujeres accedan a cargos dentro de las organizaciones de seguridad previamente reservadas para los hombres y la promoción de mujeres a los cargos máximos de las estructuras de seguridad.

De manera complementaria, otra forma de propiciar una reforma al sector seguridad contemplando el enfoque de género es por medio del gender mainstreaming (o transversalización del enfoque de género). Para Naciones Unidas, la transversalización del género consiste en la integración de la perspectiva de género en todas las etapas de los procesos de política pública (del sector seguridad): diseño, aplicación, supervisión y evaluación, con miras a promover la igualdad de género. Sin embargo, la concepción e interiorización de este eje ha tendido a ser más complicado, pues no es claro para los hacedores 
de políticas ni para el personal mismo de las fuerzas de seguridad cómo estructurar e implementar políticas de transversalización.

El enorme desarrollo normativo que existe en la actualidad frente a la necesidad de alcanzar una igualdad real entre hombres y mujeres, de prevenir violencias basadas en género, de establecer nexos claros entre las necesidades de las mujeres y la seguridad, entre otros, ha conducido a que la comunidad de Estados donantes al desarrollo y participantes activos en procesos de construcción de paz enfaticen la necesidad de generar reformas de la seguridad hacia escenarios de mayor respeto y garantía de los derechos de las mujeres y otros grupos tradicionalmente desfavorecidos. De allí que países como Colombia sean susceptibles a las influencias de estas normatividades globales, ya sea en relación con los Derechos Humanos o mediante la adopción de enfoques diferenciales y de género de cara a la construcción de paz.

La evolución de la inclusión del enfoque de género en la fuerza pública de Colombia ha sido notable, pero también sigue siendo incompleta. Si bien desde los años setenta ya existían lineamientos dentro del sector seguridad y defensa para generar políticas de discriminación positiva hacia las mujeres ${ }^{6}$, solo desde los ańos 2000 se comenzó a presenciar una verdadera proliferación de estrategias, protocolos y políticas para involucrar el tema de género en un sector que tradicionalmente había sido dominado por los hombres (Enloe, 1989; Sjoberg \& Via, 2010).

Como casi cualquier otro ejemplo a nivel mundial, las Fuerzas Militares y de Policía colombianas nacieron con la participación exclusiva de personal masculino, mientras que las mujeres se encontraban relegadas al ámbito administrativo y al cumplimiento de funciones de cuidado. Su presencia en el teatro de operaciones era casi nula, limitándose a ser el "último recurso" cuando se producían bajas masculinas. La evolución de esta situación no se dio como tal con la promulgación de normativas en pro de la inclusión, al menos no en un comienzo, sino a través de la lucha y perseverancia de las mujeres por acceder a estos ámbitos, dominados por prácticas culturales hostiles hacia ellas. La evolución de las sociedades en función de reivindicar el rol femenino en dife-

6 Mediante el Decreto 2129 de 1976 se permitió el ingreso de 12 mujeres al escalafón militar en la categoría de oficial del cuerpo administrativo del Ejército Nacional. 
rentes espacios fue lo que viabilizó y formalizó la necesidad y obligatoriedad de incluir a las mujeres con igualdad de oportunidades. Esto se hace claro en la medida en que se comienzan a verificar una serie de declaraciones a nivel internacional que abogan por la igualdad de derechos y oportunidades entre los individuos, entre las cuales se destaca la Resolución 1325 sobre Mujer, Paz y Seguridad del Consejo de Seguridad de Naciones Unidas. En ese sentido, Colombia adoptó estas normativas como parte de la suscripción de acuerdos internacionales, a raíz de los cada vez más claros procesos de globalización, y a la par de la evolución que se iba dando en el plano del sector seguridad y defensa en el mundo occidental.

$\mathrm{Al}$ igual que la adopción de la normativa de Derechos Humanos dentro de la fuerza pública colombiana, el tema de género también ha tenido que recorrer un espinoso camino en términos de su interiorización dentro del sector seguridad y defensa. Si bien la inclusión de mujeres dentro de la fuerza pública se fue haciendo paulatinamente más frecuente, esto no equivale a decir que las fuerzas de Colombia hayan adoptado un enfoque de género. En realidad, lo primero puede haber obedecido más al cumplimiento de la normativa nacional e internacional sobre la obligatoriedad de permitir el acceso de las mujeres a diferentes espacios, incluido el teatro de operaciones militares y policiales, que a una consciente y abierta aceptación de las brechas existentes y a la necesidad de abordarlo de manera integral.

Un importante impulso al tema de género se dio durante el desarrollo de los diálogos de paz entre el gobierno y las FARC. Al respecto, Huber y Karim (2017) sugieren que la finalización de los conflictos producen una oportunidad clave para crear puntos de entrada a reformas del sector seguridad con una aproximación de género. Las autoras argumentan que a partir de los diálogos abiertos que tienen lugar en la esfera pública, muchos asuntos de género, que estaban invisibilizados durante el conflicto bajo el lente de la confrontación armada, comienzan a tener cabida y a ser discutidos con miras a desarrollar políticas, estrategias y acciones.

En efecto, desde el año 2016 se registró la consolidación de espacios y plataformas de diálogo y discusión para reflexionar en torno al papel de la mujer en el posacuerdo, las cuales fueron fomentadas por organizaciones de 
mujeres y con ayuda de cooperación internacional. En este proceso fue importante la inspiración en experiencias internacionales como Guatemala, Liberia o Irlanda del Norte, donde las mujeres se organizaron políticamente y dieron viabilidad a los respectivos acuerdos de paz, además de que dejaron establecidas ciertas disposiciones de participación paritaria en la transición.

Por su parte, la mesa de negociaciones incluyó de manera explícita una subcomisión de género, la cual funcionó como una especie de comité asesor que se encargaba de revisar que todo lo que se acordara contuviera disposiciones de género. Además de ello, las partes negociadoras estuvieron en diversos escenarios con diálogos abiertos con muchas mujeres, víctimas y expertas en género, lo que ponía de presente el interés que tenía tanto el gobierno como las FARC en reconocer el papel de las mujeres en lo que estaba por acordarse.

El importante impulso que tuvo el tema de género y la inclusión femenina en los espacios públicos durante las negociaciones repercutió directamente en las fuerzas de seguridad del país. Estos espacios de diálogo y la presencia de actores internacionales que favorecieron la profundización del tema de género como condición indispensable para la construcción de paz sirvieron para darle un segundo aire a todo el proceso de inclusión que ya se estaba dando dentro de las fuerzas. Esto ocurrió especialmente desde 2010, cuando se creó la "Política en Derechos Sexuales y Reproductivos, Equidad y Violencia Basada en Género, Salud Sexual y Reproductiva, con Énfasis en VIH”, y se dio cumplimiento a las resoluciones 1325 (2000), 1820 (2008), 1888 y 1889 (2009), 1960 (2010) del Consejo de Seguridad de la Organización de las Naciones Unidas suscritas en el tema "Mujer, Seguridad y Paz" (Camacho \& Contreras, 2012).

Un ejemplo de ello fue la creación de la Oficina de Género dentro del Ejército Nacional en 2016, cuyo propósito central ha sido ajustar el tema de la inclusión femenina en las Fuerzas Armadas acorde con los protocolos internacionales. En términos prácticos, se observa que esta oficina se consolidó con el fin de mantener informadas a las fuerzas sobre los marcos normativos nacionales e internacionales relacionados con el tema de la mujer. De manera similar, la adhesión a la campaña HeforShe de Onu Mujeres en marzo de 2017 es otra muestra del compromiso de las fuerzas por fomentar la protección de los derechos de las mujeres y garantizar la igualdad de género. 
Sin embargo, pese al mayor énfasis que se ha prestado al enfoque de género, lo que se aprecia a partir de estas iniciativas desarrolladas por las instituciones de seguridad es que no existe una real comprensión de qué es el género ni de cómo se diferencia del sexo. Gran parte de la confusión se puede deber precisamente al hecho de que la normativa y la práctica de género han entrado al país por medio de una presión e influencia internacional que no necesariamente es comprendida por estas instituciones y que las ha conducido, erróneamente, a considerar que alimentar sus filas con mujeres equivale a tener una perspectiva de género.

Esta dificultad también se puede deber a otros factores. Por una parte, ya se comentó que comprender en qué consiste la aplicación del enfoque de género, por medio de las estrategias de transversalización, es una realidad que afecta tanto a hacedores de políticas, como a los operadores de seguridad en el territorio. Por otra parte, como sugieren Huber y Karim (2017), con ocasión de la transición del conflicto al posconflicto, los recursos tienden a ser limitados, incluso con la presencia de veedores y cooperantes internacionales. Esta escasez de recursos conduce a que los gobiernos, incluso aquellos que tienen la voluntad política de hacerlo, se limiten a implementar medidas de gender balancing y no de transversalización. Aún más, las resistencias de sectores de la sociedad y de las mismas fuerzas (al ser instituciones tradicionalmente hipermasculinas) puede incluso frenar la adopción de políticas integrales de género.

Pese a esto, en 2018 se consolidó la llamada "Política pública sectorial de transversalización del enfoque de género para el personal uniformado de la Fuerza Pública”, gestada por el mismo Ministerio de Defensa, bajo la dirección del exministro Luis Carlos Villegas durante la administración Santos. A todas luces, este plan de acción es de fundamental importancia porque nunca en la historia del país se había operativizado de tal modo una política integral de género que no igualara el género a lo femenino, y por lo tanto que fuera un poco más allá de la discriminación positiva hacia las mujeres.

La política se nutre fuertemente de influencias internacionales, como la Cuarta Conferencia Mundial sobre la Mujer celebrada en Beijing en 1995, por medio de la cual se establece el concepto de transversalización. Por otra parte, pero en estrecha relación con la anterior, la política se desprende de la 
apuesta del Consejo Social y Económico de la ONU (Ecosoc) de incentivar a que todas las políticas y programas del sistema de Naciones Unidas adoptaran una perspectiva de género. Finalmente, pero no menos relevante, la política se nutre claramente de lo establecido en la Convención sobre la Eliminación de Todas las Formas de Discriminación contra la Mujer (CEDAw), cuyo núcleo gira en torno a la idea de que la máxima participación de las mujeres en todas las esferas, en igualdad de condiciones con el hombre, es indispensable para el desarrollo de un país y la causa de la paz.

La política de transversalización, a diferencia de otras predecesoras, adopta un enfoque basado en Derechos Humanos, con lo que quiere poner de presente que la definición de políticas, la planificación estratégica y la ejecución estarán permeadas por un espíritu de defensa de los Derechos Humanos, por sobre cualquier otra consideración. Esto conduce a que en el marco de la política de transversalización se haya entendido y reconocido a las mujeres y demás personas víctimas de discriminación y violencia de género como sujetos de derechos que deben contar con igualdad de oportunidades para avanzar en la construcción de sus proyectos de vida, y aportar al cumplimiento de la misión de la fuerza de la cual forman parte. Si bien esto puede parecer una obviedad, históricamente las mujeres no han contado con el mismo reconocimiento de derechos que los hombres y menos aún en la fuerza pública, por lo cual es clave implementar acciones diferenciadas que le apunten a cerrar brechas de desigualdad.

Sin embargo, más allá de perseguir la equidad por medio de la adopción de un enfoque diferencial, la política pública también adopta explícitamente un enfoque de género. Este se entiende como la existencia de un sistema de dominación que se fundamenta en procesos socio-culturales que han estandarizado diferencias biológicas de forma binaria, lo cual ha redundado en la generación de jerarquías y estereotipos que han ido en detrimento de todo aquello considerado como femenino, que no se limita a las mujeres, sino a todas las personas y comportamientos que se salen de los cánones hipermasculinos y sexistas de las sociedades y, en este caso en particular, del sector de seguridad y defensa. Esta diferenciación se ha traducido en expresiones de discriminación $\mathrm{y}$ violencia, las cuales terminan por funcionar como mecanismos de control, 
en el sentido foucaulteano, limitando los comportamientos, los cuerpos y los proyectos de vida en razón al género. En este marco, el enfoque que ha planteado la política es deconstruir esas lógicas binarias y relaciones desiguales por medio de la identificación de símbolos, prácticas, valores y representaciones que mantienen y reproducen esos órdenes sociales desiguales (Ministerio de Defensa, 2017, p. 17).

Indudablemente, la apuesta del país frente al tema de género se enmarcó en el proceso de paz con las FARC, como se comentó más arriba. De allí que haya surgido la necesidad de consolidar una política desde el Ministerio de Defensa que pusiera en práctica dicho enfoque a nivel institucional. En ese sentido, es evidente que la inserción del tema de género en Colombia ha obedecido tanto a la ratificación de tratados internacionales, como también a las sugerencias externas en materia de peacebuilding. Sin embargo, la apropiación ha sido muy diferente entre las fuerzas.

Para el estamento castrense el tema de género sigue estando muy asociado con el proceso de vinculación de personal femenino dentro de sus filas y, en alguna medida, de reconocimiento de la diversidad sexual que puede existir en su interior. En términos generales, la inserción del tema de género en las fuerzas de seguridad colombianas sigue siendo un asunto en desarrollo. Si bien la política pública de transversalización está bien orientada en términos conceptuales y operativos (en cuanto a los despliegues necesarios para darle cumplimiento), esta sigue dependiendo del voluntarismo del gobierno, de las mismas instituciones de seguridad y defensa, y de la capacidad de ciertos sectores en la fuerza pública para apalancar recursos y fomentar procesos de reforma de la mano de donantes internacionales. En la actualidad, la participación de la mujer en la fuerza pública no es el asunto en cuestión; el tema de fondo está en cómo propiciar el desarrollo y la apropiación del tema de género más allá de la capacidad y la voluntariedad de grupos muy específicos de la institucionalidad. Es claro que esto suscita enormes resistencias tanto desde dentro de la fuerza pública como en la misma esfera civil, pues en el país se ha utilizado para desinformar y generar posiciones de polarización política.

Un indicador de la falta de apropiación es la escasez de informes o documentación oficial que dé cuenta de los avances de la puesta en marcha de la 
política pública de transversalización. Es posible sugerir que la internalización del enfoque de género no solo en las fuerzas sino también en la institucionalidad no ha logrado consolidarse como tal, sino que obedece más a una necesidad de dar cumplimiento a la normatividad internacional suscrita por Colombia. A esto se puede deber la falta de comprensión de lo que implica adoptar un enfoque de género y la recurrente idea de sugerir que la fuerza pública es una fuerza respetuosa de los asuntos de género por el hecho de vincular a la mujer.

\section{Conflicto de intereses}

Los autores no reportan ningún conflicto potencial de interés en la elaboración de este capítulo.

\section{Financiación}

Este trabajo fue apoyado por el proyecto de investigación INV-EES 2975 titulado "El panorama del post conflicto en Colombia: Un acercamiento desde la gobernanza y la reforma del sector seguridad", financiado en su totalidad por la Universidad Militar Nueva Granada.

\section{Referencias}

Álvarez, C., \& Duque, F. (2020). Oportunidades para las Fuerzas Militares de Colombia en operaciones multidimensionales de mantenimiento de paz. Revista Cientifica General José María Córdova, 18(29), 87-109. https://doi.org/10.21830/19006586.542

Andrade, O. (2012). Relaciones cívico-militares en Colombia: Apuntes para un estado del arte. Revista de Análisis Internacional, 6, 145-171.

Bjarnegard, E., \& Melander, E. (2011). Disentangling gender, peace and democratization: The negative effects of militarized masculinity. Journal of Gender Studies, 20(2), 139-154. https://doi.org/10.1080/09589236.2011.565194

Brzoska, M. (2011). Security, governance, and security sector governance. En F. Labarre \& M. Tzankova (eds.), Theoretical and technical perspectives on security sector governance (pp. 29-44). IOS Press.

Camacho, C., \& Contreras, I. (2012). Observatorio de equidad de género para el proceso de incorporación y seguimiento del personal estudiantil femenino de la Esmic. 
Revista Cientifica General José María Córdova, 10(10), 187-216. https://doi. org/10.21830/19006586.234

Centro de Ginebra para la Gobernanza del Sector de Seguridad [DCAF]. (2015). Security sector reform. Applying the principles of good governance to the security sector. Geneva Centre for the Democratic Control of Armed Forces.

Cooper, N., \& Pugh, M. (2002). Security-sector transformation in post-conflict societies (Draft paper for Centre for Defense Studies, King's College, Londres). http://gsdrc.org/ docs/open/ssaj150.pdf

Crandall, R. (2002). Driven by drugs: U.S. Policy toward Colombia. Lyenne Rienner Publishers.

Enloe, C. (1989). Bananas, beaches and bases. Pandora Press.

Grabendorff, W. (2009). Limited security sector reform in Colombia. En H. Born \& A. Schnabel (eds.), Security sector reform in challenging environments (pp. 69-88). Geneva Center for Security Sector Governance. https://www.dcaf.ch/sites/default/files/publications/ documents/YEARBOOK_2009.pdf

Huber, L., \& S. Karim. (2017). The internationalization of security sector gender reforms in post-conflict countries. Conflict Management and Peace Science, 35(3), 263-279. https://doi.org/10.1177/0738894217696228

Hudson, V., Ballif-Spanvill, B., Caprioli, M., \& Emmett, C. (2013). Sex and world peace. Columbia University Press.

Isacson, A. (2005). The U. S. Military in the war on drugs. En C. Youngers \& E. Rosin (eds.), Drugs and democracy in Latin America (pp. 15-60). Lyenne Rienner Publishers.

Isacson, A. (2016). El Ejército Colombiano enfrenta incertidumbre después del conflicto. Washington Office on Latin America (WoLA). http://www.wola.org/es/comentario/el_ ejercito_colombiano_enfrenta_incertidumbre_despues_del_conflicto

Kalmanovitz, P. (2018). Entre el deber de protección y la necesidad militar: Oscilaciones del discurso humanitario en Colombia, 1991-2016. Latin American Law Review, 1, 33-60. https://doi.org/10.29263/lar01.2018.02

Leal Buitrago, F. (2002). La seguridad nacional a la deriva: Del Frente Nacional a la Posguerra Fría. Alfaomega.

Marcella, G., \& Schulz, D. (1999). Colombia's three wars: U. S. Strategy at the crossroads. U. S. Army War College.

Ministerio de Defensa Nacional. (2017). Política pública sectorial de transversalización del enfoque de género para el personal de la Fuerza Pública. Ministerio de Defensa.

Morales, M., \& Tickner, A. (2019). ¿Qué sector seguridad necesita Colombia? Dilemas de la gobernanza de la seguridad y la defensa en el posconflicto. FESCOL.

Organización para la Seguridad y la Cooperación en Europa [Osce]. (2016). Security sector governance and reform. Guidelines for OSCE Staff. Osce.

Ramsey, R. (2008). From El Billar to Operation Fenix and Jaque: The Colombian Security Forces Experience, 1998-2008. Combat Studies Institute Press. 
Schnabel, A., \& Ehrhart, H.-G. (eds.). (2005). Security sector reform and post-conflict peacebuilding. Unu Press.

Sjoberg, L., \& Via, S. (2010). Gender, war, and militarism: Feminist perspectives. Aвс-Cuıo.

Tickner, A. (2000). Tensiones y consecuencias indeseables de la política exterior estadounidense en Colombia. Colombia Internacional, 49-50, 39-61. https://doi.org/10.7440/colombiaint49-50.2000.02

Tickner, A. (2007). Intervención por invitación. Claves de la política exterior colombiana y de sus debilidades principales. Colombia Internacional, 65, 90-111. https://doi.org/10.7440/ colombiaint65.2007.04

Tickner, A. (2014). Colombia, the United States and cooperation by proxy. Washington Office on Latin America (Wola). https://www.wola.org/analysis/colombia-the-united-states-and-security-cooperation-by-proxy/

Tickner, A. (2016). Exportación de la seguridad y politica exterior de Colombia. Fescol. https://library.fes.de/pdf-files/bueros/kolumbien/12773.pdf

Tickner, A. \& Morales, M. (2015). Cooperación dependiente asociada. Relaciones estratégicas asimétricas entre Colombia y Estados Unidos. Colombia Internacional, 85, 171-205. http://dx.doi.org/10.7440/colombiaint85.2015.06

Tokatlian, J. G. (1988). National security and drugs: Their impact on Colombian-US Relations. Journal of Interamerican Studies and World Affairs, 30(1), 133-160. 